\title{
Alternative method for concentration retrieval in differential optical absorption spectroscopy atmospheric gas pollutant measurements
}

\author{
Fabián A. Videla, Daniel C. Schinca, and Jorge O. Tocho
}

\begin{abstract}
Differential optical absorption spectroscopy is a widely used technique for open-column atmospheric-gas pollution monitoring. The concentration retrieval is based on the fitting of the measured differential absorbance through the Lambert-Beer law. We present an alternative method for calculating the gas concentration on the basis of the proportionality between differential absorbance and differential absorption cross section of the gas under study. The method can be used on its own for single-component analysis or as a complement to the standard technique in multicomponent cases. The performance of the method for the case of cross interference between two gases is analyzed. The procedure can be used with differential absorption cross sections measured in the laboratory or taken from the literature. In addition, the method provides a criterion to discriminate against different species having absorption features in the same wavelength range. 02003 Optical Society of America

OCIS codes: $\quad 010.0010,010.1120,280.0280,280.1120,300.0300,300.1030$.
\end{abstract}

F. A Videla (Departemento Fisicomatematica, Facultad de Ingeneria), D. C. Schinca, and J. O. Tocho (Departemento de Fisica, Facultad de Ciencias Exactas), are with the Universidad Nacional de La Plata and Centro de Investigaciones Opticas, CC124 C P:B1902 WAB, La Plata, Argentina. F. A. Videla's e-mail address is fabianv@ciop.unlp.edu.ar.

Received 14 August 2002; revised manuscript received 5 February 2003.

0003-6935/03/180001-09 $\$ 15.00 / 0$

C 2003 Optical Society of America ing slit) placed in the focal plane of the spectrometer. Thus the slit repetitively scanned a short portion of the spectrum where the gas shows absorption features. Consecutives scans are performed, up to 100 scans per second. For scans of less than $10 \mathrm{~ms}$ the effect of atmospheric turbulence is small, because its frequency spectrum is important only around $0.1-1$ Hz. In this way, single and multispecies atmospheric UV-visible absorption spectra could be recorded.

After that, Axelsson, Edner, and co-workers ${ }^{4-6}$ used a similar DOAS configuration to measure the concentrations of ozone, sulfur dioxide, nitrogen dioxide, ammonia, and some volatile organic compounds in urban environments over one kilometer path length. Bonasoni, Giovanelli and coworkers $^{7-9}$ used a linear photodiode array instead of the photomultiplier, measuring several pollutant gases in both urban and field scenarios, as well as for vertical tropospheric column.

In all of these cases, the concentration is derived either by correlation with laboratory-calibrated absorbance spectra or with synthesized spectra from a previously measured absorption cross section. The algorithm for concentration retrieval relies then on a least-squares fit of the measured differential absorbance spectrum with the reference spectrum. The main point in the DOAS method is the use of the so-called differential cross section, defined as the part 
of the absolute cross section that varies rapidly with wavelength.

In this paper we present an alternative method for determining gas concentration in DOAS measurements, using the linear relationship between the area of the measured differential absorbance curve and that of the differential absorption cross-section curve, as taken from the literature. The method can also be used as a complement to the traditional leastsquares fit, helping to discriminate among other absorbing gases in a fixed-wavelength range. The case of single- and multiple-component species is analyzed, and a comparison with the standard method is presented. The paper shows experimental results for both cases obtained in a gas test chamber and compares them with concentration values derived from calibrated electrochemical measurements.

\section{Theoretical Background}

A thorough discussion of the DOAS method can be found in the work by Platt and Platt and Perner. ${ }^{11}$ The latter tutorial contains details about basic principles, hardware, and algorithms, recommended for the interested reader. Following it briefly, the absorption of radiation is described by Lambert-Beer's law:

$$
I(\lambda)=I_{0}(\lambda) \exp [-L c \sigma(\lambda)]
$$

where $I_{0}(\lambda)$ denotes the incident intensity emitted by a suitable light source and $I(\lambda)$ is the radiation intensity transmitted through a layer of thickness $L$, where the species to be measured is present at the concentration $c$. The quantity $\sigma(\lambda)$ represents the wavelength-dependent absorption cross section, which is characteristic of each species. The determination of the light path length $L$ is usually trivial. Once these latter quantities are known, the average trace-gas concentration $c$ can be calculated from the measured ratio $I(\lambda) / I_{0}(\lambda)$ :

$$
c=\frac{\log \left[\frac{I_{0}(\lambda)}{I(\lambda)}\right]}{\sigma(\lambda) L}=\frac{A(\lambda)}{\sigma(\lambda) L},
$$

where $\mathrm{A}=\log \left(I_{0} / I\right)$ denotes the absorbance of the layer of length $L$. Typical absorbance values recorded in DOAS applications range from $10^{-2}$ to $10^{-4}$. DOAS makes use of the characteristic absorption features of trace-gas molecules along a path of known length in open atmosphere. The problem of determining the true intensity $I_{0}(\lambda)$, as would be received from the light source in the absence of any extinction, is solved by working with the so called differential absorption, which is defined as the part of the total absorption cross section of any molecule rapidly varying with wavelength in a given interval. Figure 1 illustrates this fact for the case of sulfur dioxide $\left(\mathrm{SO}_{2}\right),{ }^{12}$ but a similar concept can be applied to the case of other pollutant gases, such as nitrogen dioxide $\left(\mathrm{NO}_{2}\right)$ and ozone $\left(\mathrm{O}_{3}\right)$. As a general fact, the

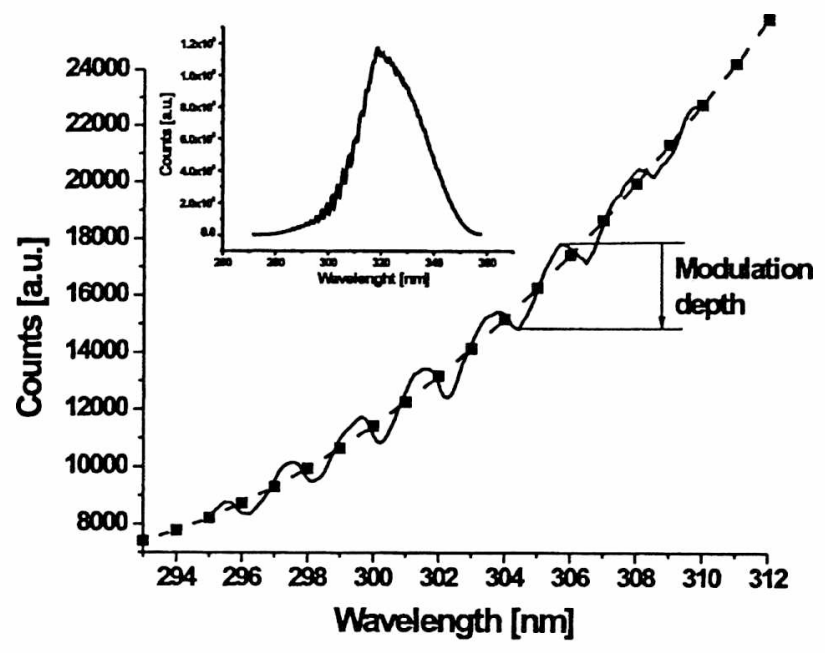

Fig. 1. Part of the raw spectra (solid curve) with interpolating third-degree polynomial (dashed curve). Inset: Typical raw spectra showing the $\mathrm{SO}_{2}$ absorption modulation in the $300-\mathrm{nm}$ region.

absorption cross section of a given molecular species may be written as the sum of two terms:

$$
\sigma_{i}(\lambda)=\sigma_{i 0}(\lambda)+\sigma_{i}{ }^{\prime}(\lambda),
$$

where $\sigma_{i 0}(\lambda)$ represents the monotonously (slow) variation of the cross section with wavelength (due to scattering, for example) and $\sigma_{i}{ }^{\prime}(\lambda)$ represents the rapid variation due to an absorption band. Although the absorption spectra of polyatomic molecules may be complicated owing to the presence of numerous closely spaced vibrational-rotational levels, ${ }^{13}$ typical spectrographs used in DOAS do not have the necessary resolving power to discriminate the rovibronic bands. So, the meaning of "slowly" and "rapid" are related to the instrument resolution and to the extent of the wavelength range. Thus a differential absorbance can be defined similarly to Eq. (2) as:

$$
A^{\prime}(\lambda)=\log \left[I_{0}{ }^{\prime}(\lambda) / I(\lambda)\right]=c L \sigma_{i}{ }^{\prime}(\lambda),
$$

where $I_{0}{ }^{\prime}(\lambda)$ is the intensity in the absence of differential absorption. ${ }^{11}$ Thus the concentration can be calculated from Eq. (4) with the $\sigma_{i}{ }^{\prime}(\lambda)$ taken from the literature (or measured in the laboratory) and fitting $I_{0}{ }^{\prime}(\lambda)$ by a suitable polynomial, up to the fifth or sixth order, depending on the selected spectral range., ${ }^{2,5}$ After we divide the raw spectrum by this polynomial and take its logarithm, a differential absorbance spectrum (or differential transmission spectrum) is obtained. We can synthesize this spectrum by multiplying $\sigma_{i}{ }^{\prime}(\lambda)$ by a suitable concentration-timeslength factor [according to Eq. (4)], which is obtained by a least-squares fit.

The residual spectrum generally shows some differential features that may be due to the presence of another species having absorption features in the same spectral region as the former or may arise from 
artifacts of the algorithm. ${ }^{5,6}$ These artifacts may be introduced by inaccurate matching between the multiple-regression analysis and the actual differential absorbance spectrum, which, after subtraction, yields a residual spectrum showing relatively large structures. ${ }^{5}$ This matching process requires shifting up and down in wavelength one of the spectra for precise peak matching. ${ }^{6}$ In addition, the reference absorption spectrum must be measured with the same instrumentation or convoluted with the appropriate instrument function to ensure the same resolution for both spectra. This fact makes necessary an accurate determination of the reference concentration to get reliable absorption cross-section values.

An alternative approach is to regard Eq. (4) as a linear parametric relation between the measured differential spectra $\left[A^{\prime}(\lambda)\right]$ and the differential cross section $\left[\sigma_{i}{ }^{\prime}(\lambda)\right]$. To make this approach effective, the two functions are matched in both wavelength range and spectral resolution. In such a case, Eq. (4) may be integrated from a certain initial wavelength value to a certain final wavelength value to yield:

$$
\int_{\lambda_{i}}^{\lambda_{f}} A^{\prime}(\lambda) \mathrm{d} \lambda=c L \int_{\lambda_{i}}^{\lambda_{f}} \sigma^{\prime}(\lambda) \mathrm{d} \lambda .
$$

Physically, this equation means that the area under the differential absorbance curve is proportional to the area under the differential cross-section curve. This interpretation is in accordance with the fact that the area under an isolated absorption line remains constant regardless of its width (for a given absorber concentration). If the absorption bands of the gas under study are close enough to each other that they are blended, the above statement may not hold strictly. Nevertheless, since $A^{\prime}(\lambda)$ and $\sigma_{i}{ }^{\prime}(\lambda)$ are matched in spectral resolution $(0.1-0.3 \mathrm{~nm}$ for a typical DOAS spectrograph), Eq. (5) still holds. In fact, numerical calculations were carried out convoluting $A^{\prime}(\lambda)$ and $\sigma_{i}^{\prime}(\lambda)$ with instrument functions of different widths (covering the range mentioned above). The changes in the resulting spectra do not modify the estimated value of concentration, since the largest width of the instrument function is much less than the width of the bands. For the cases shown in this work and, in general, for almost all gas pollutants that can be measured by DOAS, the combination of band separation, width, and spectral resolution produces negligible blending of the bands. If the lower integral limit in Eq. (5) is kept fixed and the upper limit is taken as a variable parameter, a plot of the values of both integrals for different $\lambda_{f}$ will yield a linear plot whose slope is proportional to the actual concentration.

\section{Experimental Setup}

\section{A. DOAS System}

Our DOAS system has been described in detail elsewhere. ${ }^{14}$ Briefly, it consists of a 150-W Xe arc lamp placed at the focus of a Newtonian telescope. The collimated beam traverses a long atmospheric path and is collected by another telescope and focused into a spectrograph for wavelength analysis. This setup was used previously to make measurements on $\mathrm{SO}_{2}$ and $\mathrm{NO}_{2}$ at a local oil refinery. ${ }^{15}$ Since for the present measurements the light path inside the test chamber was about 1 meter, the collimating telescope was removed and the lamp was run at about $70 \%$ of its rated power.

\section{Spectrograph}

The spectrograph was the same as the one described in a previous work. ${ }^{13}$ For high-resolution spectroscopy, an echelle-based spectrograph is preferred over common grating in low order. The main reason is its inherent higher light throughput because of its short focal length. Because the echelle grating has a large groove spacing, it is used at high-order numbers, but it is necessary to provide cross dispersion to avoid an overlapping of diffraction orders. The cross disperser is simply a prism or another grating whose dispersion is at right angles with that of the echelle. The angular dispersion of the cross disperser is usually many times smaller, so the combination of the two elements gives a two-dimensional spectrum format. The cross-dispersion spectrograph used in this work is a Mechelle 900 model from Multichannel Instruments $\mathrm{AB}$ (Sweden). The exact position of any wavelength can be determined by use of ray tracing. Thus, for a given wavelength $\lambda$, the spectral order $m$ and the focal plane co-ordinates $x_{c}, y_{c}$ can be calculated. Recording a spectral image of a calibration source (such as a low-pressure mercury lamp) containing well-defined known spectral lines provides the means for fine calibration. The image has a resolution of $0.23 \mathrm{~nm}$. Although the Mechelle 900 has a built-in pre-exposure command that optimizes exposure time to prevent pixel saturation, the relative intensity ratio between the UV (300-nm) light and near-infrared $(900-\mathrm{nm})$ light of the Xe lamp imposes the use of a UV bandpass filter to cut off most of the visible-NIR Xe emission. This fact also applies to conventional grating-based spectrographs, since the stray light effectively reaching the optical sensor (usually a CCD) is large enough to create a noisy background that masks the weak UV portion of the signal.

\section{Test Chamber}

The test chamber used in these experiments was constructed at our site and simulates a portion of an industrial chimney. . It is a stainless-steel cylinder, $50 \mathrm{~cm}$ tall and $50 \mathrm{~cm}$ in diameter with internal regulated electrical heaters that can raise the temperature inside the chamber up to $250{ }^{\circ} \mathrm{C}$. It has two pairs of optical ports (25 cm long each) placed opposite to each other through which absorptiontransmission experiments can be conducted. Since the length of optical path between these ports was $1 \mathrm{~m}$, the DOAS concentration measurements are given in parts-per-million meter. It also has four other input-output ports for gas inlet, vacuum line, vacuum gauge, and electrochemical probe connector. 


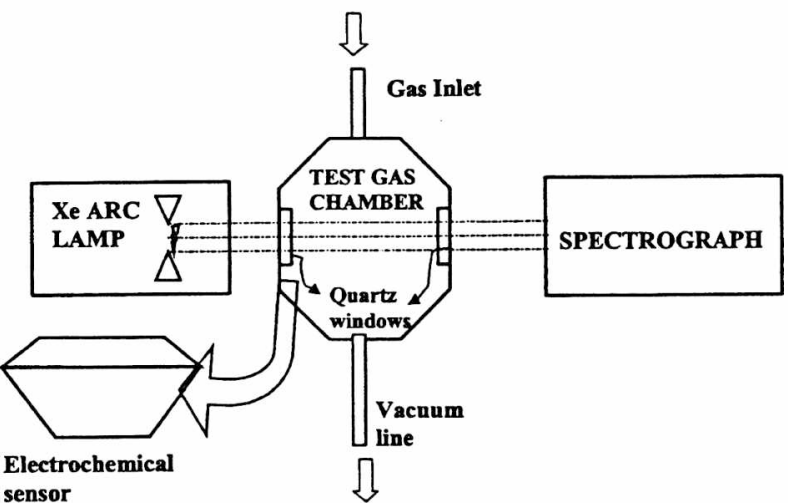

Fig. 2. Experimental setup.

The latter is a TESTO 360 electrochemical sensor capable of measuring $\mathrm{SO}_{2}, \mathrm{NO}_{\mathrm{x}}, \mathrm{CO}, \mathrm{CO}_{2}$ and oxygen in the $0-5000 \mathrm{ppm}$ range with an error of $5 \%$. An overall block diagram of the experimental setup is shown in Fig. 2.

\section{Data Processing}

Since uneven sensitivity of the CCD pixels together with a possible lack of smoothness in the intensity spectrum of the Xe arc lamp may introduce fictitious features in the absorption spectrum, a raw spectrum of the lamp in the absence of gases is recorded. A third-order polynomial is fitted and subtracted. The residual difference (positive and negative) is then subtracted from the former raw spectrum to account for the noise features. This procedure is iterated until a smooth lamp spectrum is obtained and saved for future use.

\section{A. Single Component Analysis}

The procedure is as follows: A raw spectrum is recorded as shown in the inset of Fig. 1, where it can be seen that the short wavelength side of the transmission profile of the bandpass filter is modulated by the absorption features of $\mathrm{SO}_{2}$ in the range of $280-320$ nm. Only a portion of this spectrum is taken for processing (namely, 295-310 $\mathrm{nm}$ ), since it contains several peaks usable for concentration retrieval. This spectrum is then matched to the saved smooth lamp spectrum, and the noise is subtracted to eliminate the lamp's features. The differential absorbance spectrum is evaluated according to Eq. (4), where $I_{0}{ }^{\prime}$ is a third-order polynomial and $I$ is the matched raw spectrum. At this stage, the areas are calculated and stored for values of $\lambda_{f}$ coincident with successive minima under the absorbance curve. This way of taking $\lambda_{f}$ values is for ease of calculation, but in principle all values within the wavelength range are equally selectable. An $\mathrm{SO}_{2}$ library differential cross section is selected and then convoluted with the instrumental function and its areas calculated similarly as the absorbance. This process is illustrated in Fig. 3, where part (a) shows a typical $\mathrm{SO}_{2}$ absorbance spectrum and part (b) shows a coincident portion of its cross section as taken from Brass-

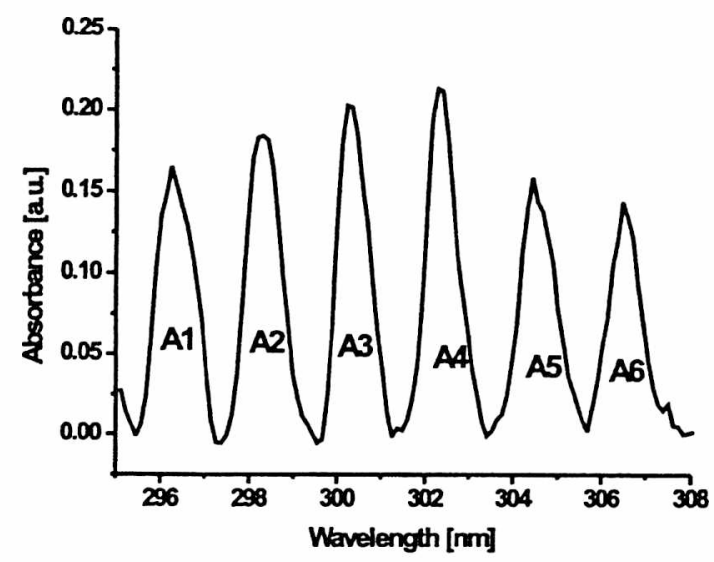

(a)

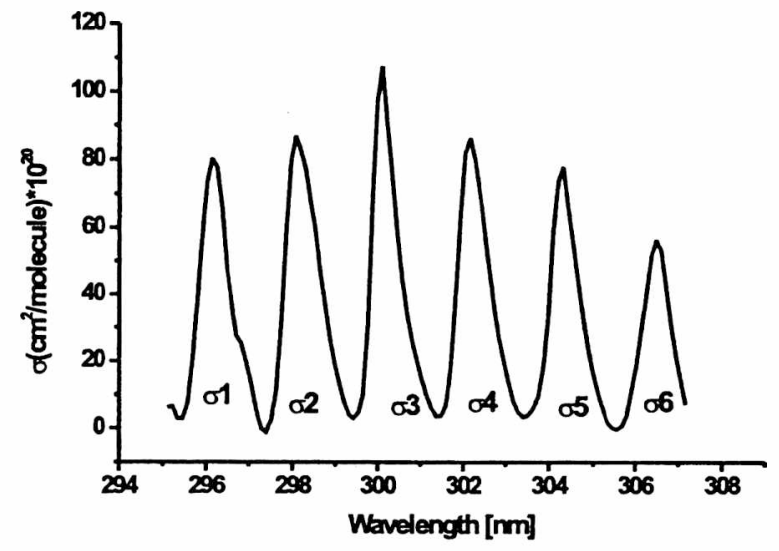

(b)

Fig. 3. (a) Typical absorbance $\mathrm{SO}_{2}$ spectrum. The labeled areas are used for concentration determination. (b) Differential absorption cross section taken from Ref. 12 convoluted with instrument function.

ington 12 convoluted with an instrument function of 0.25-nm width. In each curve, the different areas used for the calculation of Eq. (5) are labeled. Finally, the areas under each absorption band are added to yield

$$
\sum_{i=1}^{K} A_{i}=c L \sum_{i=1}^{K} \sigma_{i}{ }^{\prime}+\varepsilon_{K},
$$

where $\varepsilon_{K}$ is the error, since the left-hand side is a measured magnitude.

By varying $K$ from 1 to $n$, we obtain $n$ points that can be fitted by a linear regression whose slope is the product of $c$ times $L$ (Fig. 4). It should be noted from Fig. 3 that the index $K$ may take values from 1 to 6 (that is, several bands are taken into account), allowing a reliable regression.

\section{B. Multicomponent Analysis}

Since the total differential absorbance can be considered as the linear combination of the component differential absorbances, Eq. (5) can be generalized 


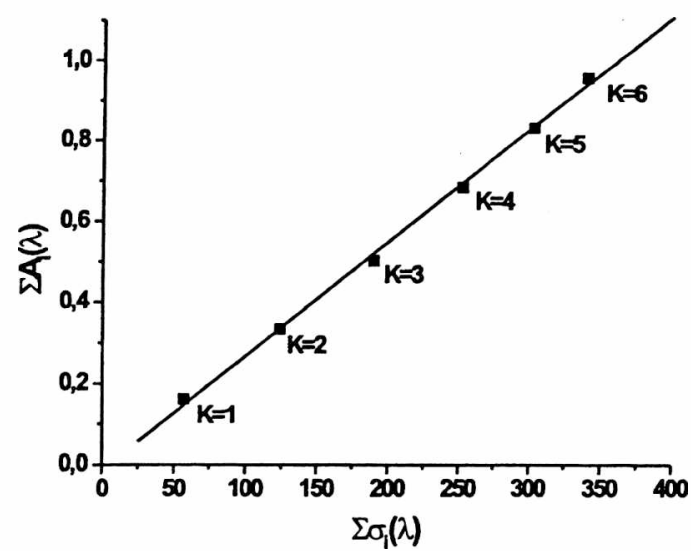

Fig. 4. Linear regression corresponding to Eq. (6). Its slope is proportional to the concentration.

to many species by rewriting its right-hand side as

$$
L \sum_{j=1}^{m} c_{j} \int_{\lambda_{i}}^{\lambda_{f}} \sigma_{j}(\lambda) \mathrm{d} \lambda,
$$

where $\sigma_{j}(\lambda)$ is the absorption cross section of species $j$, between the wavelengths $\lambda_{i}$ and $\lambda_{f} ; c_{j}$ is the number density of species $j$; and $m$ is the number of species present. For the case of two components, $m=2$, so the difference between the measured total differential absorbance value and the calculated component differential absorbances can be written as

$$
\begin{array}{r}
\int_{\lambda_{0}}^{\lambda_{f}} \operatorname{abs}(\lambda) \mathrm{d} \lambda-L\left[c_{1} \int_{\lambda_{0}}^{\lambda_{f}} \sigma_{1}(\lambda) \mathrm{d} \lambda+c_{2} \int_{\lambda_{0}}^{\lambda_{f}} \sigma_{2}(\lambda) \mathrm{d} \lambda\right] \\
=\varepsilon\left(\lambda_{f}\right),
\end{array}
$$

where $\varepsilon\left(\lambda_{f}\right)$ is the error as a function of $\lambda_{f}$. If this parameter takes $n$ values within the selected wavelength range, then Eq. (8) yields a set of $n$ equations whose solution is given in matrix form by ${ }^{16}$ :

$$
\mathbf{C}=\left[\mathbf{S}^{T} \mathbf{S}\right]^{-1} \mathbf{S}^{T} \mathbf{A}
$$

where $\mathbf{C}$ is the concentration matrix with elements $c_{11}, c_{12} . \quad \mathrm{S}$ is the $n \times 2$ matrix with elements

$$
\begin{aligned}
& s_{i 1}=\int_{\lambda_{0}}^{\lambda_{k}} \sigma_{1}(\lambda) \mathrm{d} \lambda, \\
& s_{i 2}=\int_{\lambda_{0}}^{\lambda_{k}} \sigma_{2}(\lambda) \mathrm{d} \lambda \quad k=1 . . n,
\end{aligned}
$$

where $n-1$ is the number of regions to integrate, $S^{T}$ is the transpose of $\mathbf{S}$, and $\mathbf{A}$ is the $n \times 1$ matrix with elements

$$
a_{i}=\int_{\lambda_{0}}^{\lambda_{k}} \operatorname{abs}(\lambda) \mathrm{d} \lambda .
$$

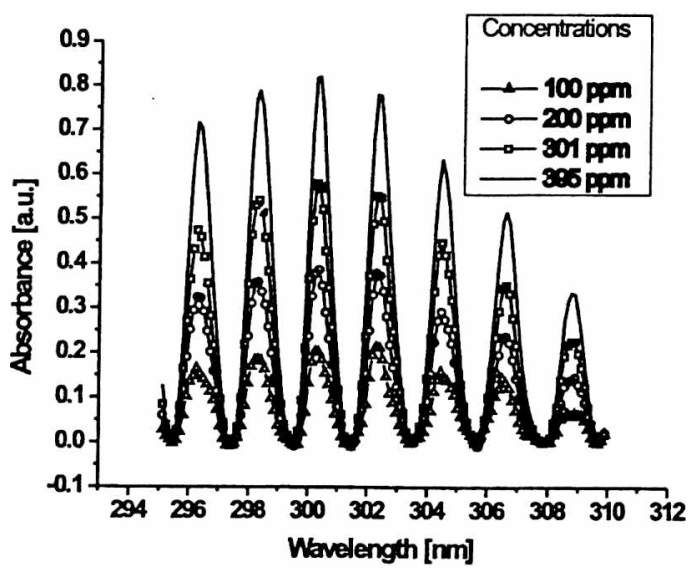

(a)

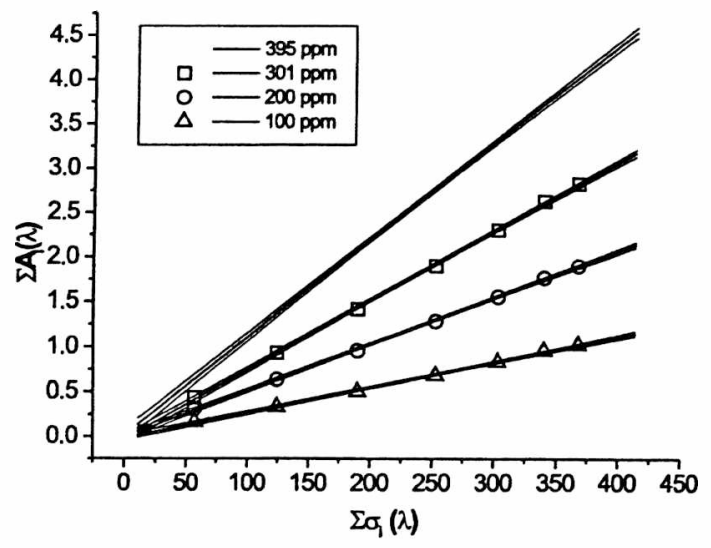

(b)

Fig. 5. (a) Differential absorbance curves for different concentration ranges. The numbers in the top right corner are the concentration measured by the electrochemical sensor. (b) Linear regression for the differential absorbance curves of part (a). The confidence interval is shown for each case. The numbers in the top left corner are the retrieved concentration values.

The use of the filter may modify the measurement of the absorbance. If the filter is placed before the absorbing media, the $1 / I_{0}$ ratio shows the actual modulation depth of the absorption features, in accordance with the Lambert-Beer law. If the filter is placed after the absorbing media (for example, at the entrance slit of the spectrograph), the modulation is modified by a factor that is a function of the filter transmission. In this case, the measured intensity values must be suitably corrected to take into account this departure from the real modulation depth.

\section{Results}

\section{A. Single-Component Case}

This procedure was tested for different $\mathrm{SO}_{2}$ concentrations diluted in $\mathrm{N}_{2}$, ranging from 4 to $400 \mathrm{ppm}$ as measured with the calibrated electrochemical sensor. Figure 5(a) shows the differential absorbance spectrum corresponding to four different concentrations with a 100-ppm interval increment. It can be seen 


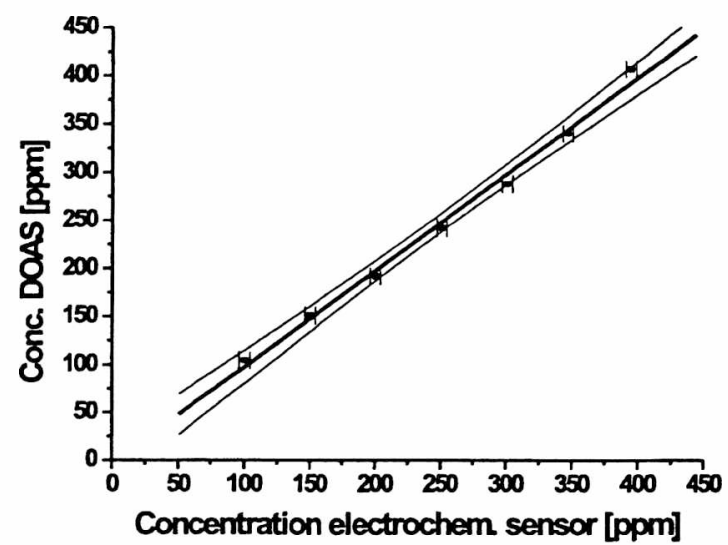

Fig. 6. Comparison between concentration values measured with electrochemical sensor and those retrieved by the integral method for single-component atmosphere.

that there is proportionality between the height of the peaks and the concentration values. If the procedure based on Eq. (6) is applied to each of these absorbance spectra, a family of linear plots is obtained [Fig. 5(b)]. The slope of each curve determines the concentration, as mentioned before. If the concentration values retrieved by DOAS are plotted against the values measured by the electrochemical sensor, it can be seen that a linear regression fits these points (Fig. 6). This shows that there is an excellent agreement between both sets of values, indicating that the integral procedure retrieves concentration values very close to those determined by nonoptical methods. Analogous results are obtained for lower concentrations (Fig. 7).

To test the method for another single gas, pure $\mathrm{NO}_{2}$ was introduced in the test chamber at different concentrations. It is well known that $\mathrm{NO}_{2}$ has its maximum differential absorption cross section in the range 430-450 $\mathrm{nm}$ and extending with decreasing intensity to both the red and the UV. For reasons that will be clear below, the wavelength range 290 $310 \mathrm{~nm}$ was chosen. A similar analysis to the $\mathrm{SO}_{2}$ may be applied to $\mathrm{NO}_{2}$. Figure 8(a) shows a typical absorption spectrum in this range for a given concentration, and Fig. 8(b) shows the corresponding linear regression.

\section{B. Multicomponent Case}

To evaluate the algorithm performance for the cross interference, several mixtures of $\mathrm{NO}_{2}$ and $\mathrm{SO}_{2}$ were tested in the chamber. The mixing ratios of these gases span 0 and $100 \%$. Figure 9 shows an example of total differential absorbance for some mixtures and the approximate mixing ratios are (a) $\mathrm{SO}_{2}: \mathrm{NO}_{2}=$ $1: 1.5$, (b) $\mathrm{SO}_{2}: \mathrm{NO}_{2}=1: 10$, and (c) $\mathrm{SO}_{2}: \mathrm{NO}_{2}=1: 30$. Use of Eq. (9) allows us to calculate the individual concentrations, whose values are shown in the bottom right corner of the figures. The dotted curves in Fig. 9 show the calculated differential absorbance with these retrieved values. It can be seen there is a good match between the measured and the calcu-

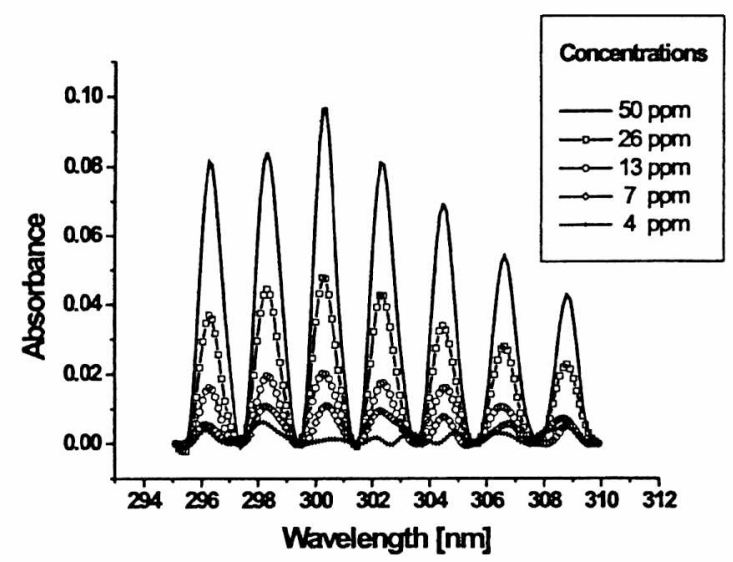

(a)

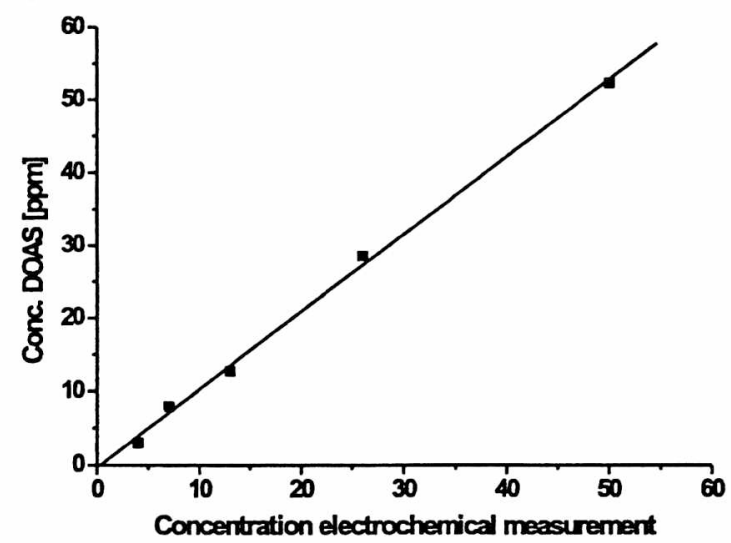

(b)

Fig. 7. (a) Differential absorbance curves for low concentration range. (b) Relation between DOAS and electrochemical sensor concentrations for the low concentration range.

lated curves. The percentual difference with the electrochemical values lies between $1 \%$ and $6 \%$. However, it was found that addition of a third gas in Eq. (9) may yield a nonnegative concentration coefficient; despite this, gas was not added to the mixture in the test chamber. This fact was checked including ozone cross section ${ }^{4,17}$ (which has a nonnegligible value in the wavelength range used). This possible drawback concerning multiple regression analysis has been mentioned by some authors. ${ }^{6}$ To overcome this problem, the integral method can be applied to the resultant spectrum obtained after subtracting from the total differential absorbance a synthesized spectrum corresponding to the component that has the largest $N$ times $\sigma$ product. Figure 10(a) shows the result of using this approach in the case of the mixture of Fig. 9(a). When a synthesized $\mathrm{SO}_{2}$ spectrum is subtracted from the total differential absorbance, a resultant spectrum is obtained (inset) that contains features of the other species plus noise. The squares correspond to $\mathrm{NO}_{2}$ and the circles to $\mathrm{O}_{3}$. Based on the proportionality between differential absorbance and concentration, it is observed that $\mathrm{NO}_{2}$ squares are best fitted by a linear regression, 


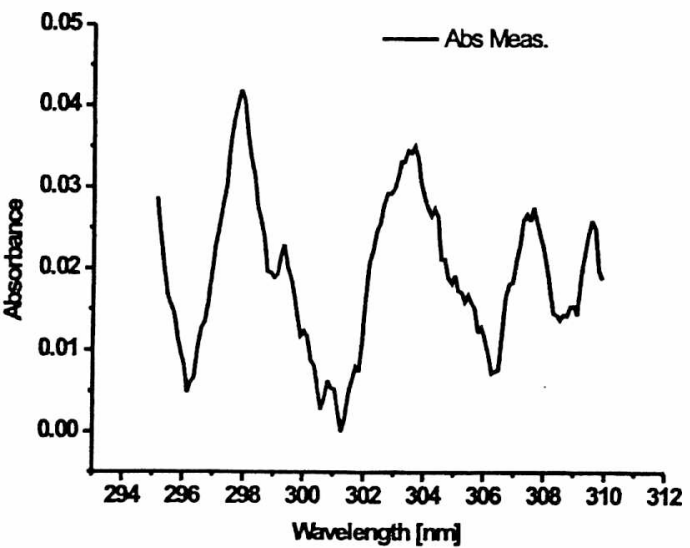

(a)

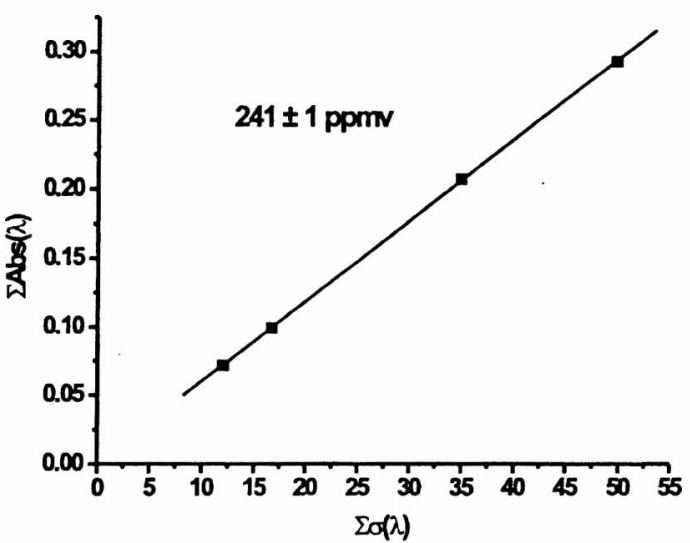

(b)

Fig. 8. (a) Differential absorbance spectrum of $\mathrm{NO}_{2}$ in the 295$310 \mathrm{~nm}$ range. The number in the top left corner is the concentration measured by the electrochemical sensor. (b) Linear regression for the differential absorbance curve of part (a). The number in the top left corner is the retrieved concentration.

whereas the $\mathrm{O}_{3}$ circles show a significant departure from linearity. This establishes a criterion to identify the possible absorbing species present. Figures $10(\mathrm{~b})$ and 10 (c) show similar results for other concentration ratios.

Finally, Fig. 11 shows the traditional least-squares method applied to the same mixing ratios used above. The dotted curves represent the calculated differential absorbances with this algorithm. Here also the numbers in the bottom right corner are the retrieved concentrations. It can be seen that the fit is very similar to the one shown in Fig. 9, and the percentual difference with the electrochemical values lies between $1 \%$ and $8 \%$. The concentration values retrieved by both methods differ from each other by about $2 \%$ to $3 \%$.

\section{Conclusions}

An alternative method for concentration retrieval in DOAS measurements on the basis of parametric integration of the differential absorbance equation is proposed. The method is tested in a laboratory gas

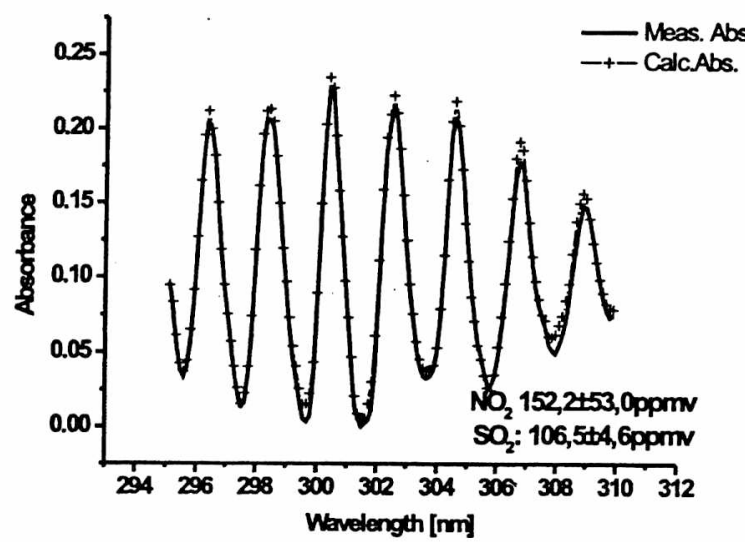

(a)

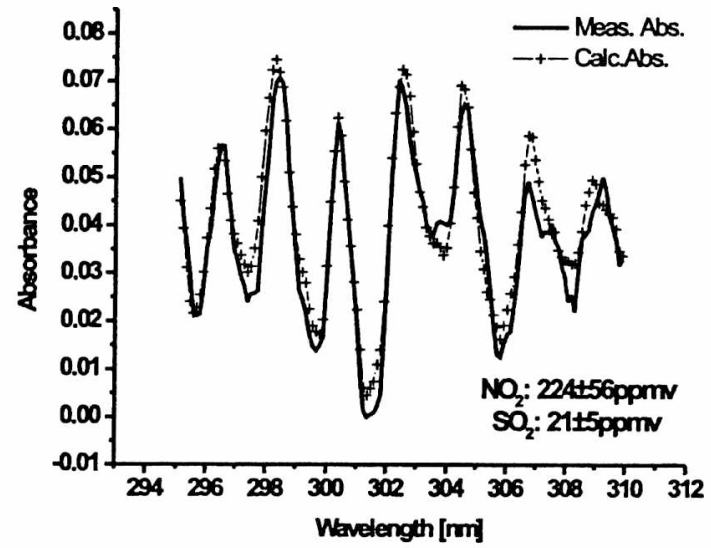

(b)

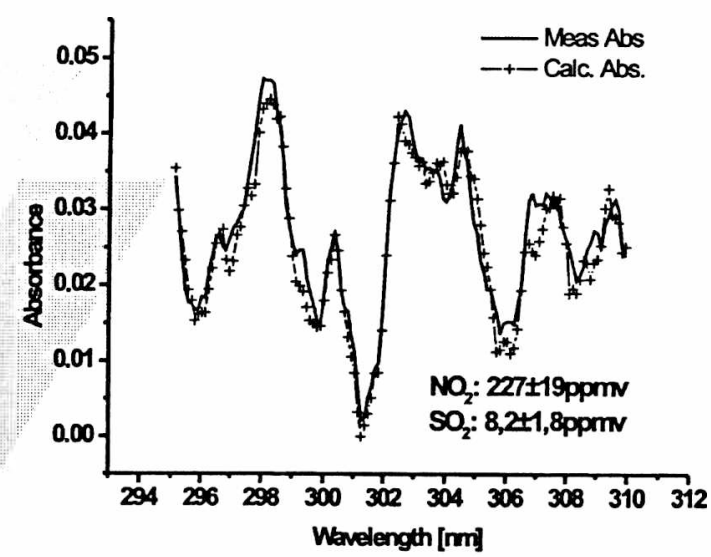

(c)

Fig. 9. Measured total differential absorbance (solid curve) and calculated differential absorbance (dotted curve) by use of the concentration coefficients determined by the integral method for different mixing ratios of $\mathrm{SO}_{2}$ and $\mathrm{NO}_{2}$ (see text). (a) $\mathrm{SO}_{2}, 105 \mathrm{ppm}$; $\mathrm{NO}_{2}, 161 \mathrm{ppm}$. (b) $\mathrm{SO}_{2}, 23 \mathrm{ppm} ; \mathrm{NO}_{2}, 216 \mathrm{ppm}$. (c) $\mathrm{SO}_{2}, 8 \mathrm{ppm}$ $\mathrm{NO}_{2}, 231 \mathrm{ppm}$.

chamber for pure $\mathrm{SO}_{2}$ and $\mathrm{NO}_{2}$ in the range of 4-400 ppm over a 1-m optical path. The retrieved concentrations agree with the values measured by a calibrated electrochemical sensor. 


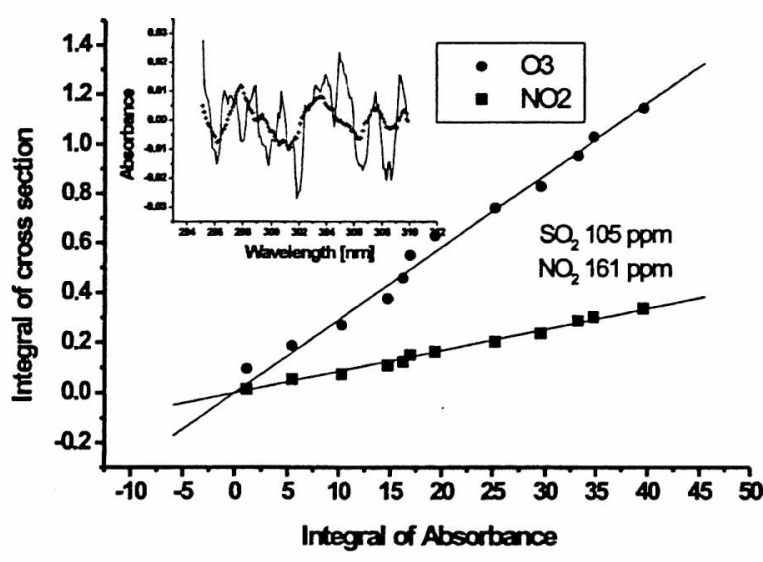

(a)

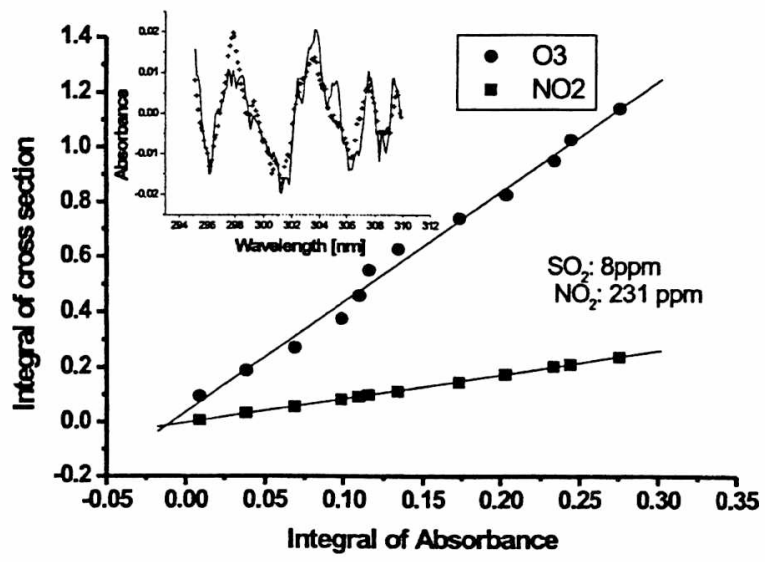

(b)

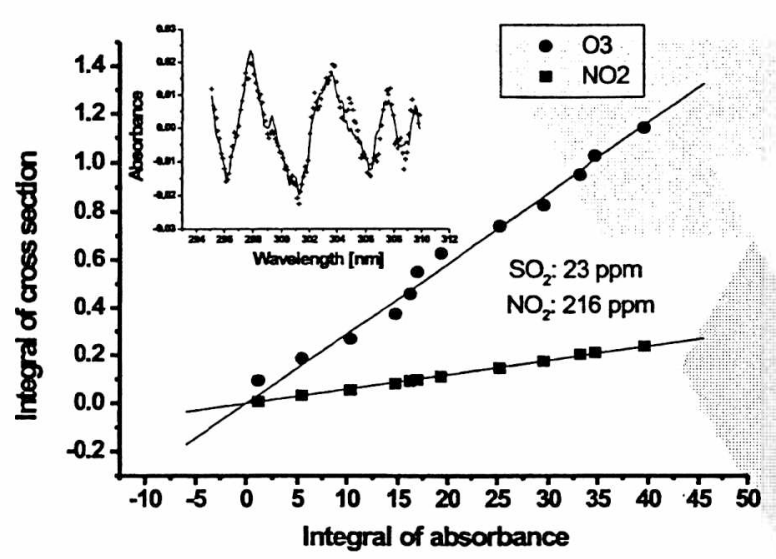

(c)

Fig. 10. Linear regression of the values calculated applying Eq. (5) to the resultant spectrum (inset) for the mixtures (a) $\mathrm{SO}_{2}, 105$ ppm; $\mathrm{NO}_{2}, 161$ ppm. (b) $\mathrm{SO}_{2}, 23 \mathrm{ppm} ; \mathrm{NO}_{2}, 216 \mathrm{ppm}$. (c) $\mathrm{SO}_{2}, 8$ ppm; $\mathrm{NO}_{2}, 231 \mathrm{ppm}$.

To expose the method to the cross-interference problem, mixtures of these gases with different mixing ratios were also measured. A multipleregression analysis applied to the values determined by Eq. (6) yields concentration that agrees with the

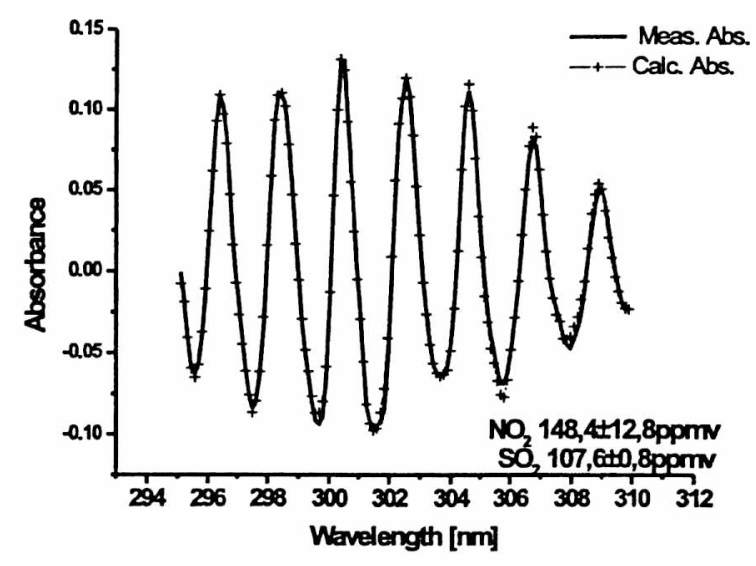

(a)

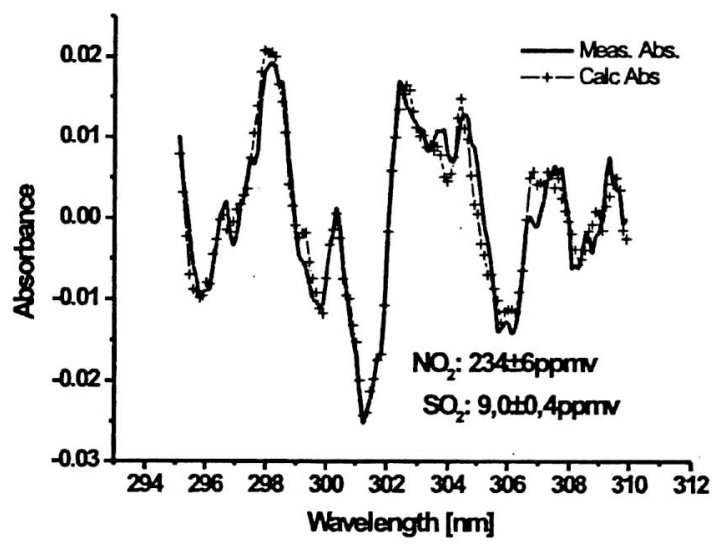

(b)

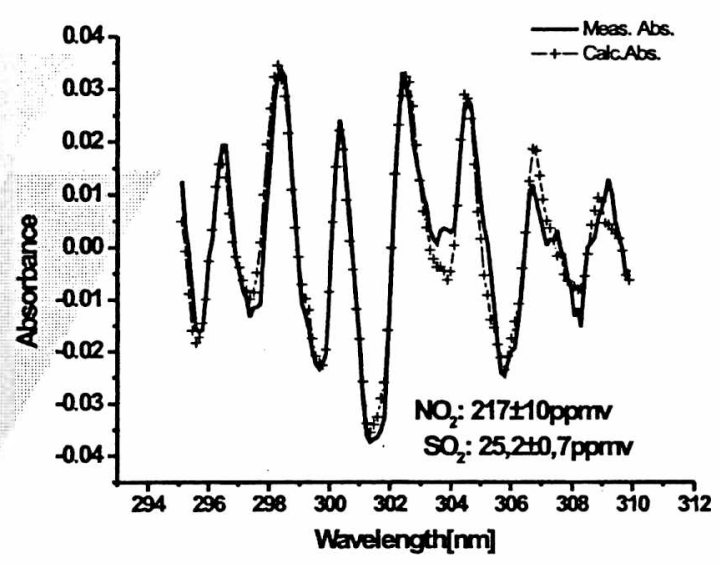

(c)

Fig. 11. Measured total differential absorbance (solid curve) and calculated differential absorbance (dotted curve) by use of the standard least-squares method for different mixing ratios of $\mathrm{SO}_{2}$ and $\mathrm{NO}_{2}$ (see text). (a) $\mathrm{SO}_{2}, 105 \mathrm{ppm} ; \mathrm{NO}_{2}, 161 \mathrm{ppm}$. (b) $\mathrm{SO}_{2}, 23$ ppm; $\mathrm{NO}_{2}, 216$ ppm. (c) $\mathrm{SO}_{2}, 8$ ppm; $\mathrm{NO}_{2}, 231 \mathrm{ppm}$.

electrochemical sensor values. Once the resultant spectrum is obtained by subtraction of the main component, the method provides a criterion to identify other species that are probably present in the mixture that absorb in the same wavelength range. 


\begin{tabular}{|l|l|l|l|l|l|l|}
\hline rich2/lp-osa/lp-osa/lp0603/lp8004-03a & reedj & $\mathrm{S}=3$ & $3 / 25 / 03$ & $13: 27$ & Art: & Input-1st mke-s, 2nd low \\
\hline
\end{tabular}

\section{References}

1. D. Perner and U. Platt, "Detection of nitrous acid in the atmosphere by differential optical absorption," Geophys. Res. Lett. 6, 917-920 (1979).

2. U. Platt and D. Perner, "Direct measurements of atmospheric $\mathrm{CH}_{2} \mathrm{O}, \mathrm{HNO}_{2}, \mathrm{O}_{3} \mathrm{NO}_{2}$ and $\mathrm{SO}_{2}$ by differential optical absorption in the near UV," J. Geophys. Res. 85, 7453-7458 (1980).

3. U. Platt, D. Perner, and H. Pätz, "Simultaneous measurements of atmospheric $\mathrm{CH}_{2} \mathrm{O}, \mathrm{O}_{3}$, and $\mathrm{NO}_{2}$ by differential optical absorption," J. Geophys. Res. 84, 6329-6335 (1979).

4. H. Axelsson, H. Edner, B. Galle, P. Ragnarson, and M. Rudin, "Differential optical absorption spectroscopy (DOAS) measurements of ozone in the 280-290 nm wavelength region," Appl. Spect. 44, 1654-1658 (1990).

5. H. Edner, P. Ragnarson, S. Spännare, and S. Svanberg, “Differential optical absorption spectroscopy (DOAS) system for urban atmospheric pollution monitoring," Appl. Opt. 32, 327333 (1993).

6. H. Axelsson, A. Eilard, A. Emanuelsson, B. Galle, H. Edner, P. Ragnarson, and H. Kloo, "Measurement of aromatic hydrocarbons with the DOAS technique," Appl. Spect. 49, 1254-1260 (1995).

7. F. Evangelisti, A. Baroncelli, P. Bonasoni, G. Giovanelli, and F. Ravegnani, "Differential optical absorption spectrometer for measurement of tropospheric pollutants," Appl. Opt. 34, 27372744 (1995).

8. G. Giovanelli, P. Bonasoni, F. Evangelisti, and F. Ravegnani, "Ozone ground-based measurements by the GASCOD near UV and visible DOAS system," in Proceedings of the Quadrennial
Ozone Symposium 1992, R. D. Hudson, ed. (Goddard Space Flight Center, Greenbelt, Md., 1992), pp. 707-710.

9. P. Bonasoni, F. Evangelisti, G. Giovanelli, and F. Ravegnani, "Remote sensing DOAS system for measurements of atmospheric trace gases," Phys. Med. 9, 304-307 (1993).

10. U. Platt and D. Perner, "Measurements of atmospheric trace gases by long path differential UV/visible absorption spectroscopy," in Optical and Laser Remote Sensing Techniques, D. K. Killinger and A. Mooradian, eds., (Springer-Verlag, Berlin, 1983), pp. $\bullet \bullet \bullet-\bullet$.

11. U. Platt, "Differential optical absorption spectroscopy," in Monitoring by Spectroscopy Techniques, M. Sigrist, ed. (Wiley,

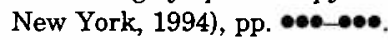

12. D. J. Brassington, "Sulfur dioxide absorption cross-section measurements from $290 \mathrm{~nm}$ to $317 \mathrm{~nm}$," Appl. Opt. 20, 37743779 (1981).

13. G. Herzberg, Electronic Spectra of Polyatomic Molecules (Van Nostrand Reinhold, New York, 1966).

14. D. Schinca and J. Tocho, "Development of a DOAS system based on a cross dispersion spectrograph," in Water, Ground, and Air Pollution Monitoring and Remediation, T. Vo-Dinh and R. Spellicy, eds., Proc. SPIE 4199,

15. D. Schinca, R. Di Paolo, F. Videla, E. Rodríguez, and J. Tocho, Medidas en Planta de $\mathrm{SO}_{2}$ y $\mathrm{NO}_{2}$ en la Región de La Plata, (Asociación Física Argentina, Argentina, 1999).

16. E. Lloyd, ed., Handbook of Applicable Mathematics, (Wiley, New York, 1984), Vol. VI, Part A, Chap. 8.

17. L. T. Molina and M. J. Molina, "Absolute absorption cross sections of ozone in the 185- to 350-nm wavelength range," J. Geophys. Res. 91, 14501-14508 (1986). 